\title{
Collaborative billiARds: Towards the Ultimate Gaming Experience*
}

\author{
Usman Sargaana ${ }^{1}$, Hossein S. Farahani ${ }^{2}$, Jongweon Lee $^{3}$, Jeha Ryu ${ }^{2}$, \\ and Woontack Woo ${ }^{1}$ \\ ${ }^{1}$ GIST U-VR Lab., Gwangju 500-712, S. Korea \\ \{sargaana, wwoo\} @gist.ac.kr \\ http://uvr.gist.ac.kr \\ ${ }^{2}$ GIST HuManCom Lab., Gwangju 500-712, S. Korea \\ \{hossein, ryu\}@gist.ac.kr \\ http: / /dyconlab.gist.ac.kr \\ ${ }^{3}$ Dept. of Digital Contents, Sejong University, \\ Seoul 143-747, S. Korea \\ jwlee@sejong.ac.kr
}

\begin{abstract}
In this paper, we identify the features that enhance gaming experience in Augmented Reality (AR) environments. These include Tangible User Interface, force-feedback, audio-visual cues, collaboration and mobility. We base our findings on lessons learnt from existing AR games. We apply these results to billiARds which is an AR system that, in addition to visual and aural cues, provides force-feedback. billiARds supports interaction through a vision-based tangible AR interface. Two users can easily operate the proposed system while playing Collaborative billiARds game around a table. The users can collaborate through both virtual and real objects. User study confirmed that the resulting system delivers enhanced gaming experience by supporting the five features highlighted in this paper.
\end{abstract}

\section{Introduction}

Augmented Reality (AR) supplements user's perception of real world by stimulating one or more senses [2]. Besides medical, military and manufacturing applications, AR is now also being used for entertainment purposes. Many of such applications are computer games. In this regard, much work has been done in research labs and we are yet to find a commercially launched AR game. However, the underlying technology has matured to an extent that AR games will soon grab a significant chunk of the entertainment industry.

It has been claimed, with convincing facts, that AR is capable of overpowering other technologies in computer gaming [1]. For this, a model of gaming experience comprising of physical, mental, social and emotional aspects was considered. It was shown that AR can exploit all the four aspects and thus provides an ideal platform for computer games. But, before the dream of commercial AR games becomes a reality,

\footnotetext{
* This work was supported by GIST.

F. Kishino et al. (Eds.): ICEC 2005, LNCS 3711, pp. 361 - 371, 2005.

(C) IFIP International Federation for Information Processing 2005
} 
several challenges must be met. Most of these challenges are related to availability of affordable devices with the required performance. With the ongoing fast-paced development in the computing hardware industry, widespread popularity of AR games is foreseeable in the near future. Besides hardware, several other issues are involved in success of computer games, application functionality being an important one. In the following, we enlist various application features that enhance a player's gaming experience in AR environment. In doing so, we do not consider the hardware constraints which are unavoidable in every AR system. Also, we exclude those aspects which are key features of AR, i.e. augmentation, interaction and registration [2]. It is assumed that all AR games cater these core requirements. Instead, we focus on functionality of gaming applications. The idea is to use the application features in a constructive fashion, with strengths of available functionality making up for the hardware constraints. These features may not be applicable to some applications, but they are general enough to be incorporated into most games. While the hardware has limitations, human imagination is unlimited. If provided enough stimulation during game-play, human imagination can go beyond all hardware constraints to perceive the ultimate gaming experience.

Tangible User Interface (TUI). TUIs provide an intuitive way of manipulating digital data through real-world objects [11]. This is because humans are naturally skilled in handling physical objects. TUIs not only provide tactile feedback, but can also map natural movements of users into the digital world. On the other hand, interaction through specialized devices is unnatural and imparts cognitive load on user in terms of learning novel ways of manipulation. ARQuake is a first-person shooting game which allows players to shoot at virtual monsters by pressing trigger of a tangible gun [3]. This improved playability of the game.

Force-Feedback. Visual cues alone are not sufficient to provide intuitive interaction to users. TUIs deliver natural tactile feedback to enhance user's experience. However, systems with TUIs still fall short of realism when dynamics is involved in a game. In such situations, force-feedback is required to ensure user's sense of realism. ARQuake used a haptic gun to shoot at virtual monsters [3]. It contains a solenoid which strikes a bolt against the gun to provide force-feedback. It only provides 1D force-feedback which is sufficient to simulate recoil of a gun. However, it is not suitable for complex scenarios.

Audio-visual Cues. Audio-visual cues are not the visual objects or sounds simply overlaid by most systems. The cues provide information about user's augmented environment and contribute to immersion. These cues must be responsive to user's actions in the game. Humans have a remarkable ability to fuse multi-modal sensory stimuli for building inferences about their surroundings. Experiments have shown that audio-visual cues affect human perception in a virtual environment by enhancing haptic sense [9].

Collaboration. One criterion for measuring gaming experience is the degree of immersion. The most effective way of immersing a user is to imitate the reality. Most of the real-world games are multi-player games which involve rich human-to-human 
interaction. In this regard, it is necessary that the multi-player gaming system does not obstruct social communication between the players. $\mathrm{AR}^{2}$ Hockey is a collaborative game that enables two players to push a virtual puck towards each other in a shared augmented space [12].

Mobility. Most games require users to move around in augmented space in order to interact with game entities. Therefore, it is important that a gaming system should not restrict mobility of the user. In addition, the system must provide untethered interaction with the game environment. It was observed during usability evaluation of ARQuake that one reason players enjoyed the game was their ability to move freely during game-play [3].

We found that not many games support all of these features. Especially, mobility and force-feedback seem to be mutually exclusive in AR games. Moreover, existing AR games fail to exploit human ability of perceiving multi-modal stimuli. We did not find any effort that employs cross-modal effects to supplement haptic perception in an AR environment. We apply all these ideas to billiARds which is an AR system that, in addition to visual and aural augmentation, provides force-feedback [10]. It provides unconstrained mobility and employs multi-modal effects for reinforcing haptic sensation in AR environment. The force-feedback is enabled by body-worn motors using wire-tension mechanism [4], [5]. Two users, carrying their own system, can easily interact with virtual balls through tangible AR interface in a collaborative environment.

This paper is organized as follows. Chapter 2 explains the design and implementation of Collaborative billiARds system. In chapter 3, we discuss experimental results. Finally, conclusions and future work are presented in chapter 4.

\section{Collaborative billiARds}

A user can play billiARds by striking augmented balls with a wooden stick. The control flow of the system is described in Fig. 1. Force-feedback is provided on the cue when a virtual ball is hit. Simultaneously, collision sound is augmented to

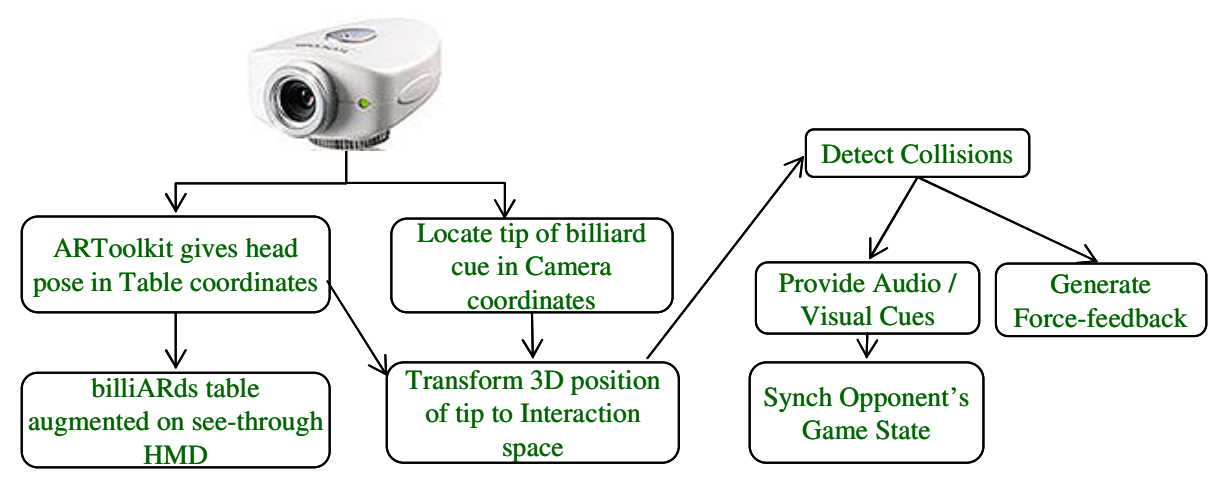

Fig. 1. Control flow of billiARds system 


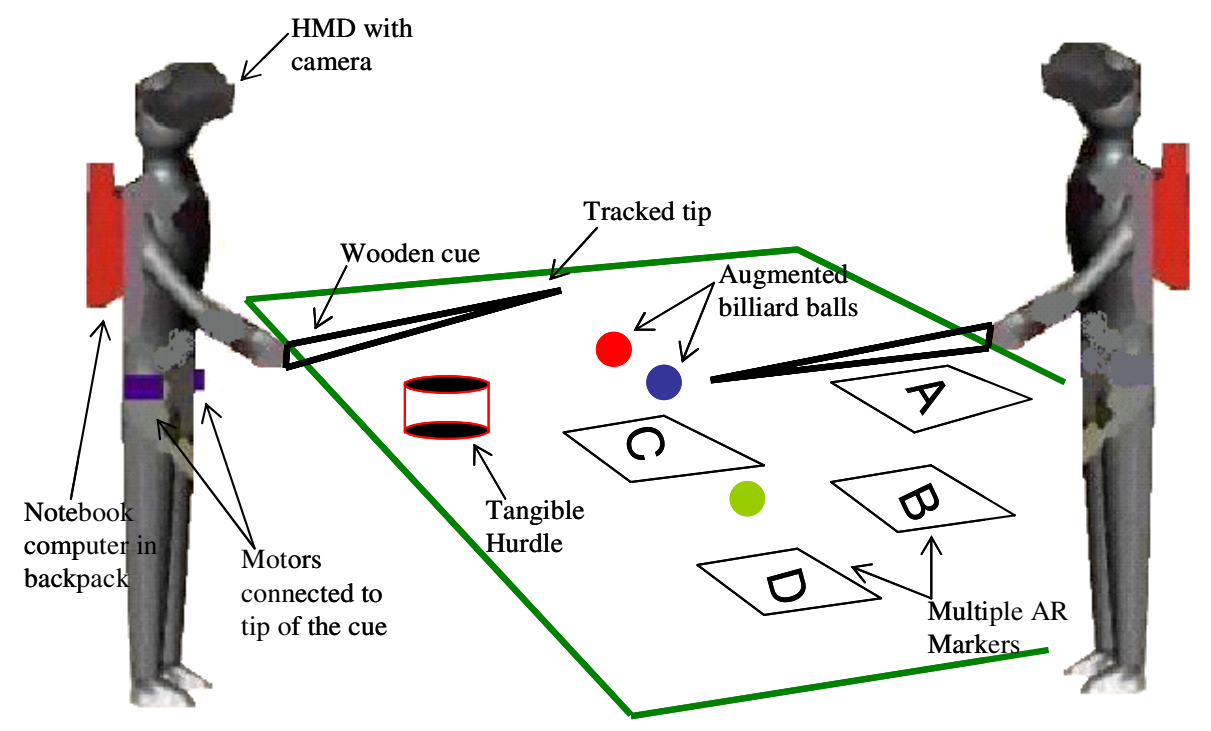

Fig. 2. System set-up for collaborative billiards

instigate user's cognition of billiards dynamics. Visual and audio cues significantly supplement user's haptic perception. Collaborative billiARds system enables two players to interact with virtual objects using tangible stick. The system set-up is illustrated in Fig. 2. In this chapter, we explain in detail the working of Collaborative billiARds system.

\subsection{Tangible AR Interface}

We develop a vision-based Tangible AR Interface for intuitive 3D interaction. We use head-mounted camera for 3D tracking of billiard cue so that user's workspace is not confined. User can operate the system while moving around in indoor environment. User interacts with game entities which can be virtual or physical object. The physical entities are in addition to the tangible cue, and can change the state of game. In the following, we explain the vision-based tracking method for tangible objects.

Tracking. We use single head-mounted camera for tracking billiard cue. For this, we attach an AR marker near the tip of the cue. ARToolkit is used to recover 6DOF pose of the billiard cue [7]. This gives a $3 \times 4$ transformation $\mathrm{T}_{\text {Cue }}$ (illustrated in Fig. 3) representing cue's position and orientation in camera coordinates. 3D position of the tip is transformed from cue coordinates to camera coordinates using equation (1).

$$
\left[\begin{array}{l}
X \\
Y \\
Z
\end{array}\right]_{\text {cam }}=\mathrm{T}_{\text {Cue }} \times\left[\begin{array}{l}
X \\
Y \\
Z
\end{array}\right]_{\text {cue }}
$$




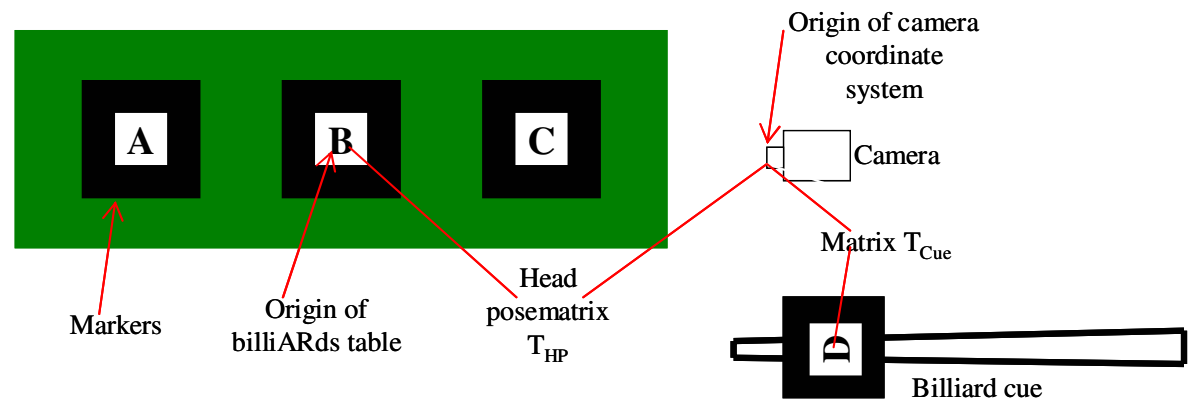

Fig. 3. Various coordinate systems

where $\left[\begin{array}{lll}X & Y & Z\end{array}\right]_{\text {cue }}^{\mathbf{T}}$ and $\left[\begin{array}{lll}X & Y & Z\end{array}\right]^{\mathbf{T}}{ }_{\text {cam }}$ denote position of the tip in cue coordinates and camera coordinates, respectively.

Interaction. We augment virtual table surface and billiard balls on a real table which bears multiple markers. The user wears a see-through HMD to view augmented objects. User's head pose (relative to the table) is required for precise augmentation and registration. This is determined by tracking multiple AR markers, placed on the table, through head-mounted camera. ARToolkit is used for this purpose. It gives us the transformation matrix $\mathrm{T}_{\mathrm{HP}}$ (illustrated in Fig. 3). The billiards table is augmented such that the virtual world coincides with marker coordinate system.

For interaction between colored tip and virtual balls, both must be placed in the same coordinate system. Tracking gives us location of the tip in camera coordinates. 3D location of the tip is transformed from camera coordinates to coordinates of augmented table using equation (2).

$$
\left[\begin{array}{l}
X \\
Y \\
Z
\end{array}\right]_{\mathrm{vt}}=\mathrm{T}_{\mathrm{HP}}^{-1} \times\left[\begin{array}{l}
X \\
Y \\
Z
\end{array}\right]_{\mathrm{cam}}
$$

where $\left[\begin{array}{lll}X & Y Z Z\end{array}\right]^{\mathbf{T}}{ }_{v t}$ and $\left[\begin{array}{lll}X & Y & Z\end{array}\right]^{\mathbf{T}}{ }_{c a m}$ denote position of the tip in virtual table coordinates and camera coordinates, respectively. In order to support interaction through wooden cue, we must detect collisions between tracked tip of the cue and virtual balls. For this, we consider radii and positions of the cue tip and the balls. Therefore, billiARds user can only strike the balls with the tip.

\section{$2.2 \quad$ Audio-Visual Cues}

When a collision between stick and a ball is detected, appropriate visual and aural cues are provided to instigate user's haptic perception. We provide four distinct auditory cues which vary in pitch and loudness according to the force of collision. The pitch and loudness of audio cues are directly proportional to the force applied by user on virtual object. In order to change the perceived pitch of the sound, we vary the sampling frequency between $45 \mathrm{kHz}$ (minimum) and $65 \mathrm{kHz}$ (maximum). Loudness perceived by the user is varied by changing intensity level of the sound. Generally, a 
$10 \mathrm{~dB}$ increase in intensity is perceived by most listeners as a doubling in loudness. We vary the intensity between the maximum (supported by the computer) to $20 \mathrm{~dB}$ less than the maximum. Table 1 shows the cues provided along with their triggering events.

Table 1. Audio Cues

\begin{tabular}{ll}
\hline Event & Duration \\
\hline When cue strikes a virtual ball. & 0.15 \\
Collision between balls. & 0.15 \\
When a ball is pocketed. & 0.37 \\
When a ball rebounds off a table rail. & 0.07 \\
\hline
\end{tabular}

billiARds Dynamics. The direction and magnitude of the push exerted by the cue on virtual ball determines the game dynamics. The magnitude of this force is proportional to how fast the cue is moved just before the strike. This is calculated from tracking history of the cue. We assume that the push exerted on the virtual ball is always directed along orientation of the cue at the time of collision. This is rational in billiards game scenario because when user hits a ball, the cue is only moved along its length. When the cue strikes a ball, we consider various variables to drive dynamics of the billiards table. These include mass and radius of balls, co-efficient of sliding friction, and velocities of colliding and deflecting balls. For balls in collision, velocities are updated using law of conservation of momentum. We observed that vision-based tracking is not accurate enough to determine the precise point on ball surface where the cue strikes. So, we make another assumption that the stick always strikes at center of the ball (center of mass). This simplifies the simulation because, as shown in equation (3), the torque becomes zero when radius and force are parallel, i.e. $\theta=0$. Thus, we ignore the angular motion of the ball and only consider translational motion.

$$
\tau=r \times F=|r||F| \sin \theta=0
$$

where $\tau$ denotes torque; and $r$ and $F$ are radius and force vectors, respectively. In order to support angular motion, i.e. spinning the ball, we need a tracking system with higher resolution and accuracy. However, such system might compromise mobility of the user or add other constraints to the system.

Occlusion. Virtual table hides part of the real scene because it is rendered over captured image of the scene. This also removes the wooden billiards cue from user's view. In order to enhance user's gaming experience, the system must deliver appropriate occlusion between real and virtual objects. Since we do not have depth map of the environment, we rely on a simpler model-based approach to handle occlusion. It works well because the only real object we deal with (i.e. billiard cue) has simple shape without any minute details. After tracking position and orientation of the real cue, we render a tapered cylinder (truncated cone) in a stencil buffer. This 


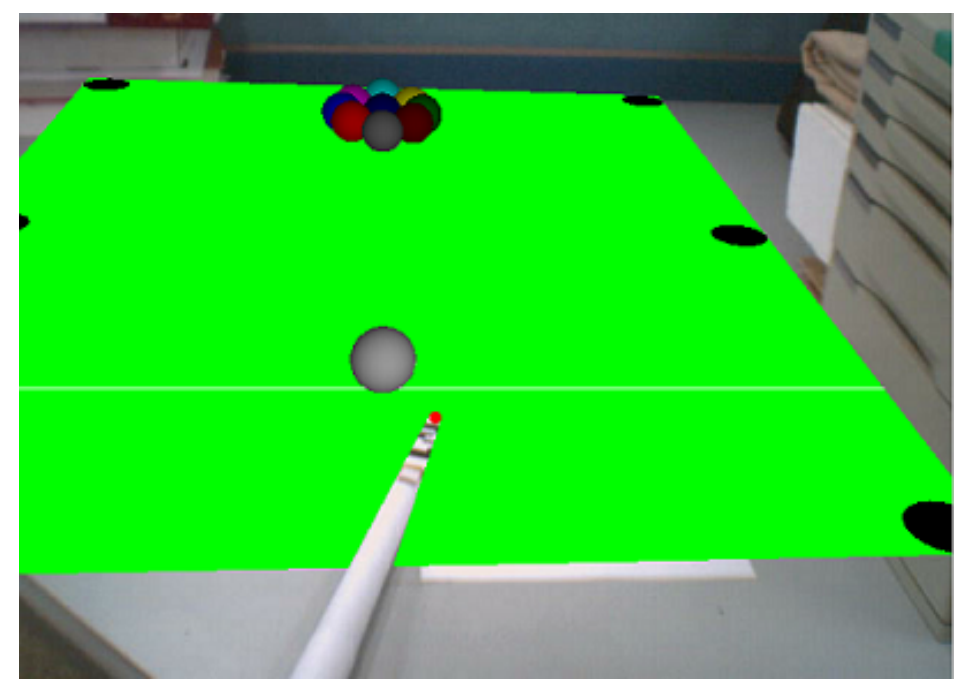

Fig. 4. Occlusion between real cue and augmented table

model is precisely rendered so that its dimensions and pose coincide with those of the real cue. When we overlay the virtual table through this stencil buffer, it covers other parts of the scene while leaving the wooden cue visible. The result is shown in Fig. 4.

\subsection{Collaboration}

Collaborative billiARds is a multi-player game that supports social interaction between two collocated users, each operating a billiARds system. Both users share the same augmented space seen through their HMDs. The system supports collaboration in two ways, i.e. through physical and virtual objects. When a user strikes the virtual cue ball at his turn, the resulting changes in the game state are transmitted to the second player using Socket communication. Both users can observe the game dynamics during the play. When all the balls come to a stop, the system notifies the player who has to strike next.

In order to keep users involved in the game, we also support collaboration through real objects. For this purpose, we slightly modified the rules of the game. In a real game, a player loses a turn or the game when a foul is committed. In Collaborative billiARds, the opponent can place tangible hurdles on the table as a penalty for a foul. Three different hurdles can be used to obstruct the opponent's strike. Each hurdle has an AR marker on top of it. The system automatically determines the placement of hurdle by detecting the marker. The updated game state is again transmitted to the other player. Shape and dimensions of all supported hurdles are known. When a hurdle is placed on table, its model is used to ensure that it is not occluded by the virtual table. This step utilizes a stencil buffer for drawing the augmented table. Furthermore, when a player sets the virtual balls in motion, the balls are now deflected by physical hurdles placed on the table. This involves collision detection which is possible because the position and model of hurdles are known. This provides 
an interesting way of collaboration through physical objects that affect state of the game. It enhances awareness of the opponent's presence and improves social interaction between players.

\subsection{Force-Feedback}

Two AC servo motors are mounted around user's waist. Each motor is attached to the billiard cue using a separate string. Using encoder data of motors, we can obtain orientation of the cue and position of the tip. While the cue is moving, the direction of motion of the tip gives us the direction of force exerted by user on the billiard ball. When the wooden cue collides with a virtual ball, a torque control algorithm for motors is used to provide force feedback. The force feedback involves producing a calculated pull, directed exactly opposite to the force applied by user, at the strings. The magnitude of force-feedback is proportional to speed of the cue before the strike. The top view of motors arrangement is shown in Fig. 5.

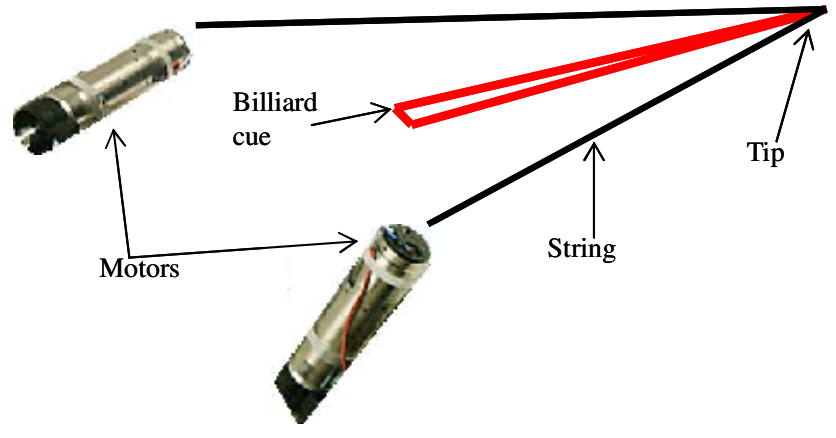

Fig. 5. Arrangement of motors for force-feedback

With two motors, the system can only provide 2D haptic sense. We observed that when a user hits a ball, the cue is almost horizontally placed for most billiard games. In such cases, the angle between cue and the table is small and the component of force perpendicular to the surface of table is negligible. Exceptions to this fact are advanced strokes, such as masse' and pique', which are played with upright cue to reverse-spin the cue-ball. These are strictly experts' shots and are rarely used by amateurs. So, we based our force reflection device on the assumption that force applied to a billiard ball is always horizontal. This assumption is rational within our game scenario, and thus, we can provide sufficient realism to the user.

In order to track the tip of the cue based on wire lengths, we solve forward kinematics. Considering reference frames $\{0\}$ and $\{1\}$, with their origins at wireextraction points, wire lengths $L_{0}, L_{l}$ can be expressed by equation (4).

$$
\begin{gathered}
L_{1}=\left\|T^{0}-S_{1}^{0}\right\| \\
L_{0}=\left\|T^{0}\right\|
\end{gathered}
$$


where $S_{1}^{0}=\left[s_{1 x}, s_{1 y}\right]$ denotes coordinates of origin of $\{1\}$ with respect to $\{0\}$, and $T^{0}=[x, y]^{\mathbf{T}}$ represents cue-tip coordinates with respect to $\{0\}$.

Solving equation (4) gives us $x$ and $y$ coordinates of the cue-tip. Unit vectors, $u_{0}$ and $u_{l}$, along the strings can be expressed by equation (5).

$$
\begin{gathered}
u_{0}=\frac{1}{\sqrt{x^{2}+y^{2}}}[x, y]^{\mathrm{T}} \\
u_{1}=\frac{1}{\sqrt{\left(x-s_{1 x}\right)^{2}+\left(y-s_{1 y}\right)^{2}}}\left[\left(x-s_{1 x}\right),\left(y-s_{1 y}\right)\right]^{\mathrm{T}}
\end{gathered}
$$

The calculation of haptic force does not depend on posture of the player. Even when a player stoops down to hit a ball, we only need position of cue-tip relative to each motor. Since the motors are mounted on a frame around waist, their relative position remains constant. We use equation (6) to calculate the tension required in wires to exert force $f$ on the cue tip.

$$
\begin{gathered}
f=p_{0} u_{0}+p_{1} u_{1}=J^{-T} p \\
J=\left[u_{0}, u_{1}\right]^{-T}
\end{gathered}
$$

where $p$ is the vector of wire tensions, and $J$ is Jacobian matrix of the haptic device. From equation (6), we can write:

$$
p=J^{T} f
$$

While the cue-tip moves freely in space, wire-tensions may become negative. This renders the wire-lengths indeterminable. Therefore, a small force is always applied at the cue-tip to ensure that the wire-tensions remain positive. To ensure transparency of haptic device, this force must be constant at all locations. Magnitude of this force depends on structure of device and user's feeling. Experimenting with different values, we found that $1 \mathrm{~N}$ is the optimal value to keep wire-tensions always positive without compromising transparency of the haptic device.

\section{Experimental Results}

We performed experiments to evaluate the role played by each of five aspects in enhancing gaming experience. Each of two players used a notebook computer (carried in backpack) with $1.5 \mathrm{GHz}$ processor, NVIDIA GeForce FX5200 graphics accelerator [8] and wireless LAN support. The users put on i-glasses SVGA head-mounted display with $800 \times 600$ pixel resolution [6]. A pair of earplugs was also provided for aural feedback. We used camera with video capture resolution of $640 \times 480$ pixels and shutter speed of 20 frames per second. A table $(60 \mathrm{~cm}$ high) was set up with seven AR Markers; each having dimensions of $8 \mathrm{~cm} \times 8 \mathrm{~cm}$. For experiments, we augmented a virtual table over an area of $100 \mathrm{~cm} \times 150 \mathrm{~cm}$. Players carried a $60-\mathrm{cm}$ long wooden cue for interaction. Force-feedback was provided with two brushless DC motors fixed to a belt around user's waist. Due to limited number of available motors, only one player had the force-feedback device. The motors can provide an output torque of 120 
$\mathrm{mNm}$. The encoders operate at 540 pulses per revolution and the encoder data can track the tip with an accuracy of $2 \mathrm{~mm}$. We mounted a camera on HMD and attached an AR Marker of size $5 \mathrm{~cm} \times 5 \mathrm{~cm}$ near the tip of billiard cue.

We conducted an informal user study during which ten different users (two at a time) put on our system and experienced the Collaborative billiARds game by moving around the table. Since players were likely to compare Collaborative billiARds with the real billiards game, we selected users who hardly ever played real billiards game. However, all these users frequently played other computer games. Six different experiments were carried out. Table 2 describes the game features provided during each setup. In experiment I, the system provided all the five features. So, we used the results of experiment I as ground basis for comparison. In rest of the experiments, the system supported all but one of the five features.

Table 2. Experiment Setup

\begin{tabular}{lccccc}
\hline $\begin{array}{l}\text { Features } \\
\text { Supported } \rightarrow\end{array}$ & $\begin{array}{l}\text { Tangible } \\
\text { User } \\
\text { Interface }\end{array}$ & $\begin{array}{l}\text { Force- } \\
\text { feedback }\end{array}$ & $\begin{array}{l}\text { Audio- } \\
\text { Visual } \\
\text { Cues }\end{array}$ & $\begin{array}{c}\text { Collaboration } \\
\text { Mobility }\end{array}$ \\
\hline Experiment I & $\sqrt{ }$ & $\sqrt{ }$ & $\sqrt{ }$ & $\sqrt{ }$ & $\sqrt{ }$ \\
Experiment II & $\times$ & $\sqrt{ }$ & $\sqrt{ }$ & $\sqrt{ }$ & $\sqrt{ }$ \\
Experiment III & $\sqrt{ }$ & $\times$ & $\sqrt{ }$ & $\sqrt{ }$ & $\sqrt{ }$ \\
Experiment IV & $\sqrt{ }$ & $\sqrt{ }$ & $\times$ & $\sqrt{ }$ & $\sqrt{ }$ \\
Experiment V & $\sqrt{ }$ & $\sqrt{ }$ & $\sqrt{ }$ & $\times$ & $\sqrt{ }$ \\
Experiment VI & $\sqrt{ }$ & $\sqrt{ }$ & $\sqrt{ }$ & $\sqrt{ }$ & $\times$ \\
where $\sqrt{\text { shows that the feature was provided, and } \times \text { denotes that the feature was not available }}$ \\
uring that experiment.
\end{tabular}

Afterwards, each user was given a questionnaire to rate the gaming experience on a 10 -scale (0-9). Only the responses from users with the force-feedback were recorded (except in Experiment III). The results of this study are compiled in Table 3. For trials done without audio-visual cues, sampling frequency and intensity of audio cues were kept constant. Also, parameters for visual cues remained constant irrespective of the force applied by user.

Table 3. User Feedback

\begin{tabular}{lll}
\hline Gaming Experience $\rightarrow$ & Mean & Std. Dev. \\
\hline Experiment I & 8.6 & 0.5 \\
Experiment II & 4.5 & 0.8 \\
Experiment III & 6.6 & 0.88 \\
Experiment IV & 7.3 & 0.3 \\
Experiment V & 7.8 & 0.91 \\
Experiment VI & 4.7 & 0.67 \\
\hline
\end{tabular}

During the experiment, we observed that the players enjoyed the game most when all the five features were provided. This observation was reinforced by the 
user-feedback data (Table 3) which exhibited that the system provided best gaming experience in Experiment I. Moreover, the results of subsequent experiments showed that removing any of the five features resulted in reduced gaming experience. This confirms that the five features we identified are indispensable for providing enhanced gaming experience in AR games. In this regard, there may exist game features other than those listed here.

\section{Conclusions and Future Work}

In this paper, we identify ways to enhance gaming experience in AR environments. We confirmed validity of the proposed ideas by applying them to Collaborative billiARds. Experiments showed that the users enjoyed the resulting gaming experience by collaborating with each other. In the future, we plan to evaluate the generality of our findings by applying them to a variety of AR games. In addition, we will develop more realistic physics model for generating audio-visual cues. Furthermore, we will study in detail the human factors involved in perception of multi-modal stimuli that instigates human imagination to perceive the ultimate gaming experience.

\section{References}

1. Nilsen, T., Linton, S., Looser, J.: Motivations for Augmented Reality Gaming. In Proc. Fuse 04, New Zealand Game Developers Conference (2004) 86-93.

2. Azuma, R. T.: A Survey of Augmented Reality. Presence: Teleoperators and Virtual Environments 6, 4 (1997) 355 - 385

3. Thomas, B. H., Krul, N., Close, B., Piekarski, W.: Usability and Playability Issues for ARQuake. In Proc. 1st Int'l Workshop on Entertainment Computing (2002)

4. Bonivento, C., Eusebi, A., Melchiorri, C., Montanari, M., Vassura, G.: WireMan: A portable wire manipulator for touch-rendering of bas-relief virtual surfaces. In Proc. 8th International Conference on Advanced Robotics (1997) 13 - 18.

5. Hirose, M., Hirota, K., Ogi, T., Yano, H., Kakehi, N., Saito, M., Nakashige, M.: HapticGEAR: The development of a wearable force display system for immersive projection displays. In Proc. IEEE Virtual Reality (2001) 123 - 129.

6. http://www.i-glassesstore.com

7. Kato, H., Billinghurst, M.: Marker Tracking and HMD Calibration for a video-based Augmented Reality Conferencing System. In Proc. 2nd International Workshop on Augmented Reality (1999)

8. http://www.nvidia.com

9. Miner, N., Gillespie, B., Caudell, T.: Examining the Influence of Audio and Visual Stimuli on a Haptic Display. In Proc. IMAGE Conference (1996)

10. Sargaana, U., Farahani, H. S., Lee, J., Ryu, J., Woo, W.: billiARds: Augmented Reality System with Wearable Force-Feedback Device. International Conference on Human Computer Interaction (Accepted) (2005)

11. Ishii, H., Ullmer, B.: Tangible Bits: Towards Seamless Interfaces between People, Bits and Atoms. In Proc. CHI '97 (1997) 234-241.

12. Ohshima, T., Satoh, K., Yamamoto, H., Tamura, H.: AR ${ }^{2}$ Hockey: A Case Study of Collaborative Augmented Reality. In Proc. IEEE Virtual Reality Annual International Symposium (1998) 268-275. 